\title{
Quantification of copper content with laser induced breakdown spectroscopy as a potential indicator of offal adulteration in beef
}

\author{
Maria Piedad Casado-Gavalda \\ Technological University Dublin, maria.casado@tudublin.ie \\ Yash Dixit \\ Technological University Dublin \\ David Geulen \\ Trier University of Applied Sciences
}

See next page for additional authors

Follow this and additional works at: https://arrow.tudublin.ie/schfsehart

Part of the Food Science Commons, Medicinal-Pharmaceutical Chemistry Commons, and the Medicine and Health Sciences Commons

\section{Recommended Citation \\ Maria P. Casado-Gavalda, Yash Dixit, David Geulen, Raquel Cama-Moncunill, Xavier Cama-Moncunill, Maria Markiewicz-Keszycka, Patrick J. Cullen, Carl Sullivan, Quantification of copper content with laser induced breakdown spectroscopy as a potential indicator of offal adulteration in beef, Talanta, Volume 169, 2017, Pages 123-129, ISSN 0039-9140, DOI: 10.1016/j.talanta.2017.03.071.}

This Article is brought to you for free and open access by the School of Food Science and Environmental Health at ARROW@TU Dublin. It has been accepted for inclusion in Articles by an authorized administrator of ARROW@TU Dublin. For more information, please contact arrow.admin@tudublin.ie, aisling.coyne@tudublin.ie, gerard.connolly@tudublin.ie.

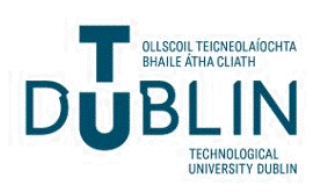




\section{Authors}

Maria Piedad Casado-Gavalda, Yash Dixit, David Geulen, Raquel Cama-Moncunill, Xavier Cama-Moncunill, Maria Markiewicz-Keszycka, Patrick J. Cullen, and Carl Sullivan 


\title{
Quantification of copper content with laser induced breakdown spectroscopy as a potential indicator of offal adulteration in beef
}

\author{
Maria P. Casado-Gavalda ${ }^{\mathrm{a}, *}$, Yash Dixit ${ }^{\mathrm{a}}$, David Geulen ${ }^{\mathrm{b}}$, Raquel Cama-Moncunill ${ }^{\mathrm{a}}$, \\ Xavier Cama-Moncunill ${ }^{a}$, Maria Markiewicz-Keszycka ${ }^{a}$, Patrick J. Cullen ${ }^{a, c}$, Carl Sullivan ${ }^{a}$ \\ a School of Food Science and Environmental Health, Dublin Institute of Technology, Dublin 1, Ireland \\ b Department of Food Technology, Trier University of Applied Sciences, Trier, Germany \\ ${ }^{\mathrm{c}}$ School of Chemical Engineering, University of New South Wales, Sydney, Australia
}

\section{A R T I C L E I N F O}

\section{Keywords:}

LIBS

Beef

Offal

Copper

PLSR

Chemometrics

\begin{abstract}
A B S T R A C T
Laser induced breakdown spectroscopy (LIBS) is an emerging technique in the field of food analysis which provides various advantages such as minimal sample preparation, chemical free, rapid detection, provision of spatial information and portability. In this study, LIBS was employed for quantitative analysis of copper content in minced beef samples spiked with beef liver over three independent batches. Copper content was determined with graphite furnace atomic absorption spectroscopy (GFAAS) in order to obtain reference values for modelling. Partial least square regression (PLSR) was performed to build a calibration and validation model. A calibration model with a high $R_{c v}^{2}$ of 0.85 and a RMSECV of $43.5 \mathrm{ppm}$ was obtained, confirming a good fit for the model. The validation model showed a good prediction accuracy with a high $R_{p}^{2}$ of 0.85 and RMSEP of $36.8 \mathrm{ppm}$. Moreover, on a further study to evaluate the spatial capabilities, LIBS was able to successfully map copper content within a pellet, indicating the suitability of LIBS to provide spatial information and therefore potential use on heterogeneous samples. Overall, it can be concluded that LIBS combined with chemometrics demonstrates potential as a quality monitoring tool for the meat processing industry.
\end{abstract}

\section{Introduction}

Meat products are very popular and important to consumers due to their high nutritional nature, as they contain a rich source of many essential trace minerals like iron, zinc and copper [1]. Amongst those, copper in lean beef is present at the lowest concentrations, in the order of $1 \mathrm{ppm}$ in raw meat. Copper is an important micromineral necessary for iron metabolism and plays an important role in the functioning of critical enzymes and the central nervous system [2,3].

As world meat production is expected to double by 2050, providing safe and hygienic meat products is of great importance [4]. Minced beef, being the main ingredient for products such as sausages, hamburger patties, meatballs and meat paste, has been targeted for adulteration with cheaper substitutes such as offal [5-8]. Offal is a rich reservoir for nutrients and as a result the mineral content of offal is typically significantly higher than in muscle tissue. In particular, copper in beef liver is found in concentrations up to 100 times higher than in lean beef $[2,3]$. Therefore, the smallest increase of copper concentration in lean beef due to liver contamination would be a good indicator for food product authenticity.
Laser-induced breakdown spectroscopy (LIBS) is a novel technique in the area of food analysis with potential as a monitoring tool for the meat industry. Emerging from laser developments in the 1960's, LIBS first saw commercialization in the field of metallurgy. Based on atomic emission spectroscopy (AES), a laser pulse ablates a small amount of material, typically a few micrograms, forming a micro plasma containing a mixture of excited neutral and charged species, both in atomic and molecular form. The light emissions from these excited species are measured as they return to their ground state using spectrophotometers [9,10]. LIBS provides various advantages such as requiring minimal sample preparation, chemical free, rapid detection, spatial information and system portability as compared to the co-existing technologies $[11,12]$.

LIBS is still in its infancy in the field of food analysis and only a few studies have been conducted with meat. Andersen et al. used LIBS for at-line monitoring of calcium content in minced poultry meat, being able to separate samples according to three calcium levels: very low ( $<$ $20 \mathrm{mg} / 100 \mathrm{~g} \mathrm{Ca}$ ), intermediate $(20-90 \mathrm{mg} / 100 \mathrm{~g} \mathrm{Ca}$ ), and high (> $90 \mathrm{mg} / 100 \mathrm{~g} \mathrm{Ca}$ ) [9]. Bilge et al. used LIBS to identify beef, pork and chicken as well as determining meat mixtures of pork adulterated beef and chicken adulterated beef [13].

\footnotetext{
* Corresponding author.

E-mail address: maria.casado@dit.ie (M.P. Casado-Gavalda).
} 
On the other hand, some studies have determined copper content in different food matrices using LIBS. Gondal et al. [14] quantified copper amongst other elements in six different pelletised tea samples using a quadrupled Q-switch Nd:YAG $266 \mathrm{~nm}$ pulsed UV laser at a maximum output energy of $30 \mathrm{~mJ}$. Copper content in the different tea samples was determined in the range of $17 \mathrm{ppm}$ and $36 \mathrm{ppm}$ with an estimated limit of detection (LOD) of $6 \mathrm{ppm}$. Sun et al. [15] determined copper content amongst other elements in powdered leaf samples fixed onto a double-sided tape by LIBS using a $100 \mathrm{~mJ}$ Nd: YAG laser at $1064 \mathrm{~nm}$ obtaining a LOD for copper of $0.08 \mathrm{ppm}$.

Most studies used chemometric techniques in order to enhance the analytical performance of LIBS. Chemometrics employs different multivariate techniques to extract useful information from large spectral data in order to perform qualitative and quantitative analysis on them [16,17]. Partial least square regression (PLSR) is a popular multivariate technique used recently to quantify mineral content in meat $[9,13]$. PLSR is a quantitative spectral decomposition technique based on linear transition from a large number of original descriptors to a new variable space based on small number of orthogonal factors called latent variables. It performs decomposition on both the spectral and concentration data simultaneously optimising the covariance between Y and linear combinations of X $[7,18,19]$.

In this study, LIBS along with PLSR modelling, was employed for the quantitative analysis of copper content in minced beef samples spiked with beef liver over three independent batches. Since copper in beef offal is reported to be in concentrations up to 100 times higher than lean beef, as it is the case of beef liver, copper was chosen as a possible indicator for liver adulteration in beef. To the best of our knowledge, no other studies involving LIBS analysis of meat have been conducted before using independent batches as part of the calibration and validation models, which is an important step in order to take into account bio-variability between animals. Moreover, the spatial capabilities of LIBS were evaluated as a potential use on heterogeneous samples.

\section{Materials and methods}

In order to ensure reproducibility of the results and take into account bio-variability between animals, all methodologies described below were conducted over three independent batches obtained on different days. For clarification purposes, these batches will further be referred as batch 1, batch 2 and batch 3 .

\subsection{Sample preparation}

Fresh beef striploin steaks and beef liver weighing approximately $200 \mathrm{~g}$ were purchased from a local butchers shop in Dublin, Ireland. A laboratory blender (8011 G, Waring Laboratory Science, Stamford CT, USA) was used to separately mince each portion, which were then transferred to disposable aluminium dishes in a drying oven for $12 \mathrm{~h}$ at $105{ }^{\circ} \mathrm{C}$. The following day, samples were further grounded into fine powder using a laboratory blender (8011G, Waring Laboratory Science, Stamford CT, USA). A series of mixtures of lean beef powder and liver powder containing $0 \%, 20 \%, 40 \%, 60 \%, 80 \%$ and $100 \%$ of liver content $(\mathrm{w} / \mathrm{w})$ were prepared for all batches with the addition of two mixtures, $30 \%$ and $70 \%$ in the case of batch 3 . A total of 60 pellets (six concentrations in triplicates for batch 1 and 2 and eight concentrations in triplicates for batch 3) containing approximately $400 \mathrm{mg}$ were prepared by thoroughly mixing $300 \mathrm{mg}$ of the prepared mixtures with $100 \mathrm{mg}$ of a binder (3644-Ultrabind, Spex, UK) with the help of a mortar and pestle. The addition of a binder helps improve the quality of the pellet by increasing the particles interaction/agglutination, and diminishing the pellets' porosity. Binders are mainly composed by hydrogen and carbon to provide mechanical strength to the pellet without interfering with the main elements in a spectrum [20]. The final mixtures were pelleted using a hydraulic press (GS01160, Specac Ltd., Orpington, United Kingdom) and a $10 \mathrm{t}$ force was applied for
$3 \mathrm{~min}$, resulting in a pellet of approximately $1.3 \mathrm{~cm}$ in diameter. In addition, two more pellets were prepared from batch 3 following the same procedure in order to obtain two pellets of approximately $400 \mathrm{mg}$ spatially occupying two different mixtures; half a side of the pellet being pure lean and the other side pure liver, and half a side of $30 \%$ liver $(w / w)$ and the other half $70 \%$ liver $(w / w)$.

\subsection{Graphite furnace atomic absorption spectroscopy analysis}

Copper content on lean beef and beef liver was determined using graphite furnace atomic absorption spectroscopy (GF-AAS) (AA240Z, Varian, USA) in order to provide reference values to build, along with the LIBS spectral data, a calibration and validation model. The standard method of AOAC (999.11-2005) was followed for sample preparation with some modifications; approximately $1 \mathrm{~g}$ of powdered sample was transferred to a crucible in triplicates and pre-ashed on a hot plate with the careful addition of small drops of purified nitric acid (CAS 7697-37-2, Sigma Aldrich, Inc.) to help digestion until completely charred. Samples were then transferred to a muffle furnace at $550^{\circ}$ for at least $4 \mathrm{~h}$ until grey/white ashes were obtained. Ashes were dissolved into $50 \mathrm{~mL}$ volumetric flasks with $1 \mathrm{M}$ nitric acid (CAS 7697-37-2, Sigma Aldrich, Inc.). A further dilution with $1 \mathrm{M}$ nitric acid was performed in order to maintain the copper concentration within the GF-AAS optimum measuring range $(0-20 \mathrm{ppb})$. Calibration curves were performed using standard solutions of copper (cat. no. 68921, Sigma Aldrich, Inc.). All samples were measured in triplicates.

\subsection{LIBS spectra acquisition}

Pellets were analysed with a LIBSCAN 150 (Applied Photonics Limited, Skipton North Yorkshire, United Kingdom), comprising of a Q-switched Nd:YAG laser operating at $1064 \mathrm{~nm}$ with pulse energy of $150 \mathrm{~mJ}$ and pulse duration of $5 \mathrm{~ns}$ (ultra, Quantel laser, 601 Haggerty Lane Bozeman, MT, USA) coupled with six fibre-optic compact optical spectrophotometers covering the wavelength range of 185-904 nm (AvaSpec Avantes spectrometers, Netherlands). The full width at half maximum (FWHM) ranged from $0.06 \mathrm{~nm}$ for the deep ultraviolet (UV) range to $0.18 \mathrm{~nm}$ for the visible near infrared (Vis-NIR) range. Light was collected by an array of 6 plasma light collection optics located in the laser head for the different wavelength regions. Additionally, the system incorporates a miniature CCD camera enabling the monitoring of the analysis from the computer screen.

Each pellet was placed in a motorised X-Y-Z sample chamber (XYZ750, Applied Photonics Limited, Skipton North Yorkshire, United Kingdom) at the optimum focal length of approximately $80 \mathrm{~mm}$. Samples were measured by scanning 100 different locations in a $10 \times 10$ grid pattern while the sample was moved by a step size of $0.70 \mathrm{~mm}$ after 3 accumulation shots per location. A repetition rate of $1 \mathrm{~Hz}$ was employed. The minimum integration time of $1.1 \mathrm{~ms}$ in Qswitched mode was chosen as an increase in integration time would not lead to a further increase of the spectrum intensity since the lifetime of the plasma is generally shorter than $1 \mathrm{~ms}$ [21]. The minimum gate delay of $1.27 \mu$ s was employed as yielded the maximum signal to noise $(\mathrm{S} / \mathrm{N})$ ratio while obtaining acceptable well-resolved spectra.

Emission spectrum for each sample were collected and further studied for pre-processing and data analysis.

\subsection{Data analysis}

All data analysis was performed using R [22] and the "pls" package [23] for PLSR (partial least square regression) modelling along with other in-house functions.

Prior to PLSR modelling, the spectral data was pre-processed in order to correct non-linearities and variation signals introduced by matrix effects and fluctuations in the laser pulse energy [24,25], which would affect the performance of the models by introducing undesired 
noise to the model. Firstly, the data collected for each pellet was averaged, obtaining a total of 18 spectra for batch 1 and 2 (6 concentrations in triplicates) and 24 spectra for batch 3 (8 concentrations in triplicates). Initial pre-processing was performed using the Standard Normal Variate (SNV) transformation to the full spectra in order to remove undesired signal variations from the acquired spectra. This transformation is a standard score calculation for each point of the signal in order to normalise any variation between point measurements within replicates [26]. Spectral data in the range of wavelengths from 316.5 to $343.2 \mathrm{~nm}$ was used for data analysis as two main emission spectral lines related to copper at $324.7 \mathrm{~nm}$ and $327.4 \mathrm{~nm}$ exists in this region [27]. The processed data was analysed and modelled using PLSR. PLSR is a multivariate technique which develops a linear regression model by projecting the predicted and observed variables to a new space to which $\mathrm{X}$ and $\mathrm{Y}$ data are transferred $[25,28]$.

In order to develop the calibration model, processed data acquired for batch 1 and batch 2 were used, along with their elemental reference values extracted from GF-AAS analysis. The data acquired from batch 3 , along with their elemental reference values, were used as the validation sample set. The method of leave-one-out was used for cross validation while developing the calibration models in order to avoid either over- or under-fitting of the models. The calibration model was evaluated by determining the optimum number of components based on the root mean square error in cross validation (RMSECV), which provides information about the deviation of the model from their reference values [29]. The corresponding values of coefficients of determination in cross validation $\left(R_{c v}^{2}\right)$ were also calculated in order to evaluate the robustness of the model. The prediction accuracy of the developed calibration model was evaluated by calculating the root mean square error of prediction (RMSEP) and the corresponding coefficients of determination in prediction $\left(R_{p}^{2}\right)$ [30]. Additionally, the limit of detection (LOD) for pseudounivariate calibration was estimated according to IUPAC official recommendations and latest development in error-in-variables theory for PLS calibration [31].

\section{Results and discussion}

\subsection{Graphite Furnace atomic absorption spectroscopy analysis}

As copper levels in beef liver are known to be richer than beef lean tissues, copper was the element chosen as an indicator of liver content. The efficiency of GF-AAS results rely heavily on the calibration curve obtained using standard solutions of the desired element. Good calibrations were obtained with a coefficient of determination for calibration $\left(R_{c}^{2}\right)$ of 0.99 . Results of GF-AAS analysis are illustrated in Table 1, showing that, as expected, copper content in lean beef is remarkable lower than in beef liver. The results presented in Table 1 were in good agreement with those reported in the literature [2] and were used as reference values to further develop the calibration and validation models.

\subsection{Spectral analysis}

Initial preliminary analysis of the different LIBS spectra was performed in order to determine the main differences in the emission of elements between the samples studied. Fig. 1(a) shows the SNV transformed LIBS spectra of lean beef and beef liver. Each spectrum corresponds to an average of 100 spectra collected per pellet. Main spectral emission lines common to both spectra corresponding to C I (247.9 nm), Mg II (279.6 nm), Zn I (330.3 nm), Ca II (393.34 nm), Ca I $(422.7 \mathrm{~nm}), \mathrm{Mg} \mathrm{I}(518.4 \mathrm{~nm}), \mathrm{Na}$ I $(588.9 \mathrm{~nm}$ and $589.6 \mathrm{~nm}), \mathrm{H} \mathrm{I}$ $(656.3 \mathrm{~nm}), \mathrm{N}$ I $(742.4 \mathrm{~nm}, 744.3 \mathrm{~nm}$ and $746.9 \mathrm{~nm}), \mathrm{K} \mathrm{I}(766.5 \mathrm{~nm}$ and $769.9 \mathrm{~nm})$, O I $(777.2 \mathrm{~nm}), \mathrm{Rb}$ I $(780.0 \mathrm{~nm})$ and $\mathrm{Na}$ I $(818.3 \mathrm{~nm}$ and $819.4 \mathrm{~nm}$ ) were observed and identified with reference to the NIST database [27]. Additionally, Fig. 1(a) clearly shows the appearance of two emission lines in the liver spectrum at around $324.7 \mathrm{~nm}$ and
Table 1

Copper content in dry matter (DM) of samples determined by GF-AAS over three independent batches.

\begin{tabular}{lll}
\hline Sample & Batch & Cu (ppm DM) \\
\hline Lean beef Powder & 1 & 4.96 \\
& & $( \pm 0.12)$ \\
& 2 & $4.84-5.08)$ \\
& $( \pm 0.26)$ \\
& $(4.65-5.10)$ \\
Beef liver powder & 3 & 6.36 \\
& & $( \pm 0.55)$ \\
& $(5.98-6.75)$ \\
& 340.81 \\
& & $( \pm 15.92)$ \\
& $(330.81-359.17)$ \\
& 333.84 \\
& 2 & $( \pm 5.78)$ \\
& 3 & $328.24-339.78)$ \\
& & $( \pm 2.92)$ \\
& & $(305.69-311.06)$
\end{tabular}

Standard deviation shown in brackets, preceded by the symbol $\pm(n=3)$.

Numbers in brackets below standard deviations correspond to the minimum and maximum values respectively.

$327.4 \mathrm{~nm}$ corresponding to $\mathrm{Cu}$ I which were not observed in the lean beef spectrum [27]. A closer inspection on the $\mathrm{Cu}$ I emission lines at $324.7 \mathrm{~nm}$ and $327.4 \mathrm{~nm}$ for all batches and liver percentages analysed is presented in Fig. 1(b) and (c) respectively. A clear increase in both emission lines with liver percentage is observed, especially a large difference in intensity between pure lean beef and beef samples containing $20 \%$ of liver $(\mathrm{w} / \mathrm{w})$ is observed, suggesting beef adulteration with liver at lower levels could be easily detected by its copper content. It should be noted that although LIBS is based on optically thin plasma emission, self-absorption of the spectral lines is a common systematic error in quantitative analysis, in which emission line intensities are not proportional to the concentration of the element in the plasma. Nevertheless, self-absorption can be minimised by the appropriate choice of gate delay time and/or selection of suitable emission lines [32]. Therefore, a range of wavelengths covering the two main $\mathrm{Cu}$ I emission lines was used for data analysis. As observed in Fig. 1(b) and (c), the LIBS spectra clearly differentiated liver adulterated beef samples based on copper content in all batches, suggesting the choice of measuring conditions was also suitable for the thin plasma model.

\subsection{Multivariate data analysis}

Chemometrics employs different multivariate techniques to perform qualitative and quantitative analysis and plays an important role extracting useful information from the large spectral data obtained in LIBS analysis [25]. Andersen et al. employed PLS modelling to predict the calcium content of minced poultry meat [9]. Similarly, Bilge et al. employed principal component analysis (PCA) to discriminate different meat species followed by PLS modelling to predict pork and chicken adulteration in beef [13]. In this study, PLSR was performed on preprocessed LIBS data in order to develop predictive models for copper content in beef as an indicator of adulteration with liver.

PLSR generates linear prediction models by optimising the covariance between the spectral data and the reference values. In order to do so, it performs decomposition on both the spectral and reference data simultaneously $[7,18]$.

\subsubsection{Development of the calibration model}

As previously mentioned, processed data acquired for batch 1 and batch 2 in the wavelength range from 316.5 to $343.2 \mathrm{~nm}$ were used, 


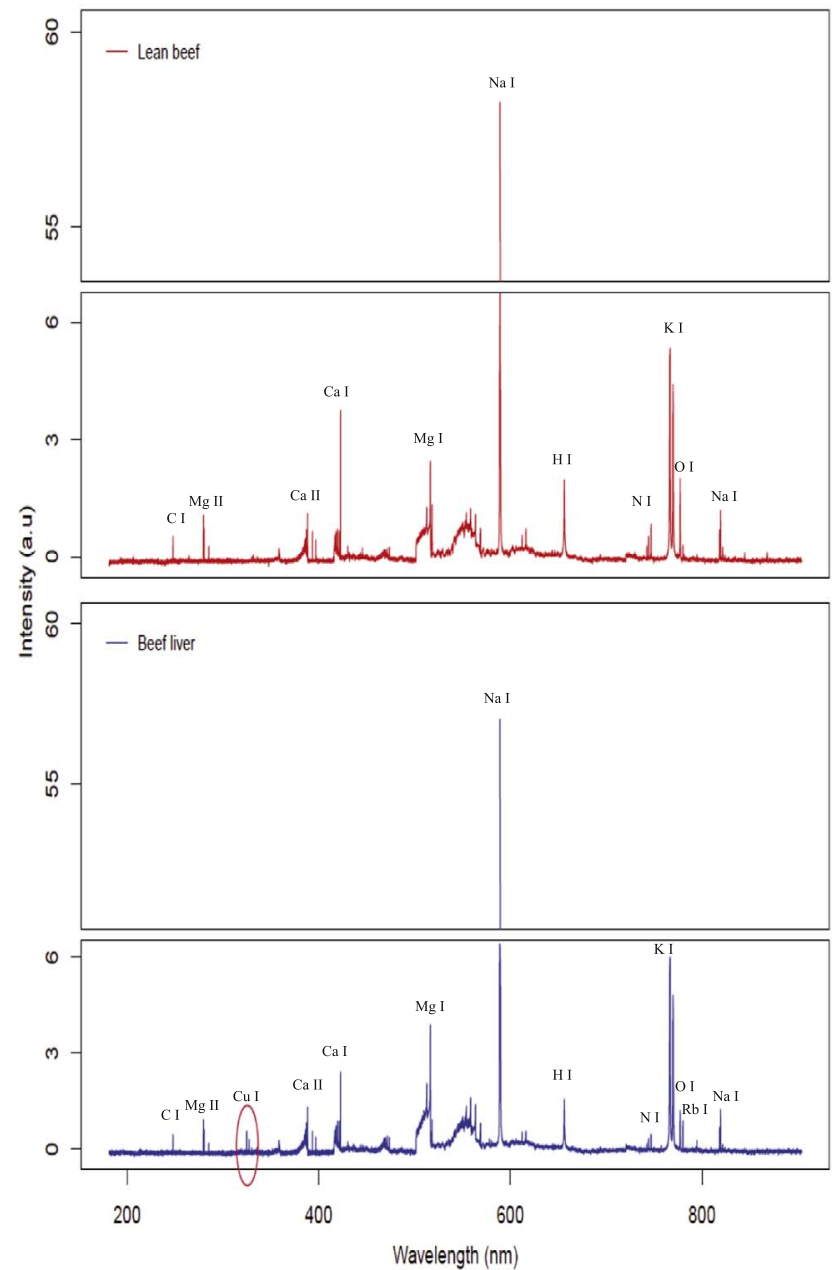

(a)

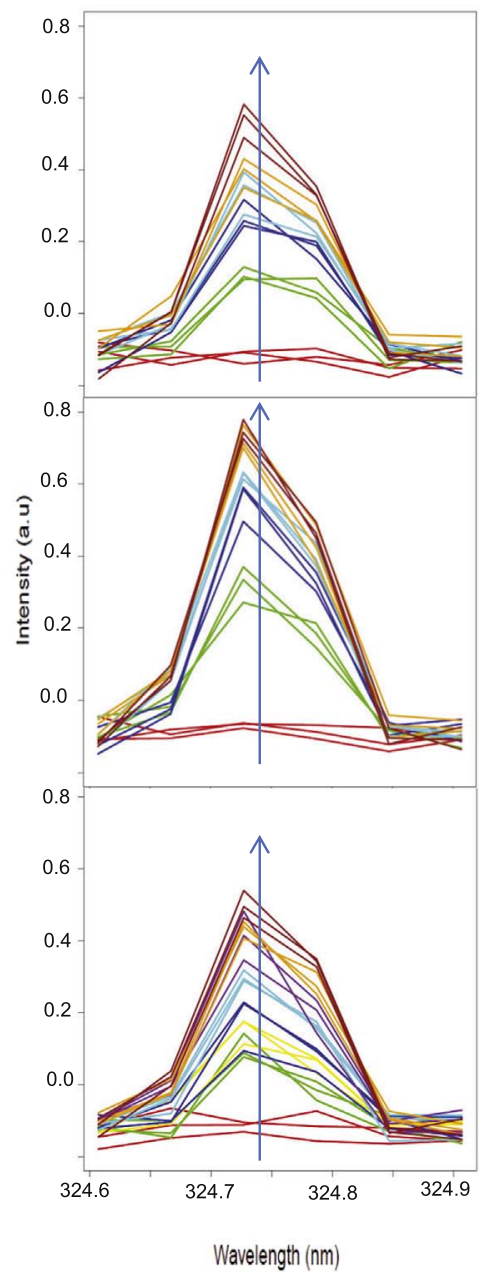

(b)

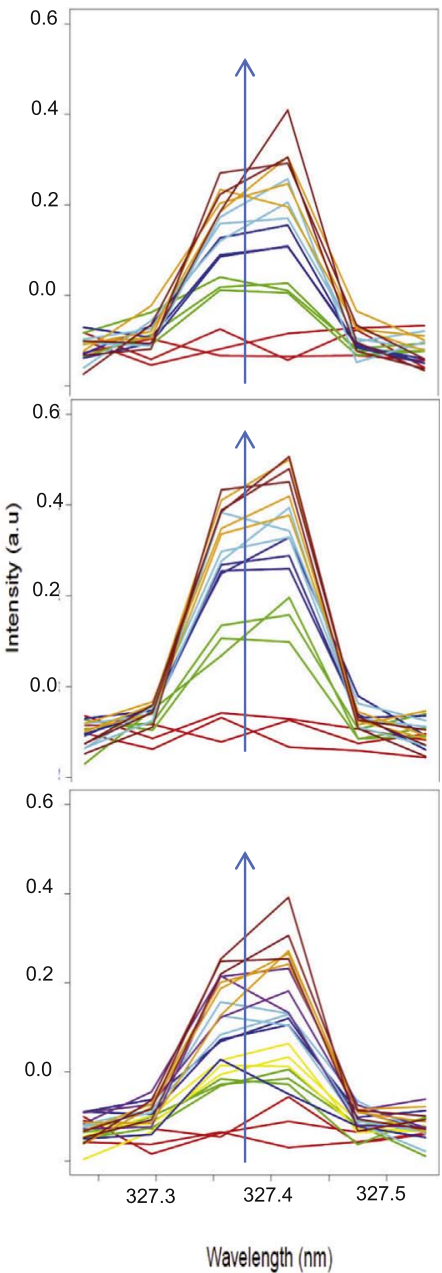

(c)

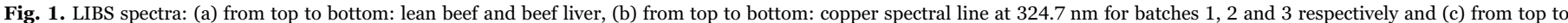

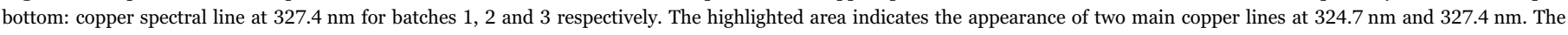
upward arrow $(\uparrow)$ indicates an increase in copper with increase of liver percentage.
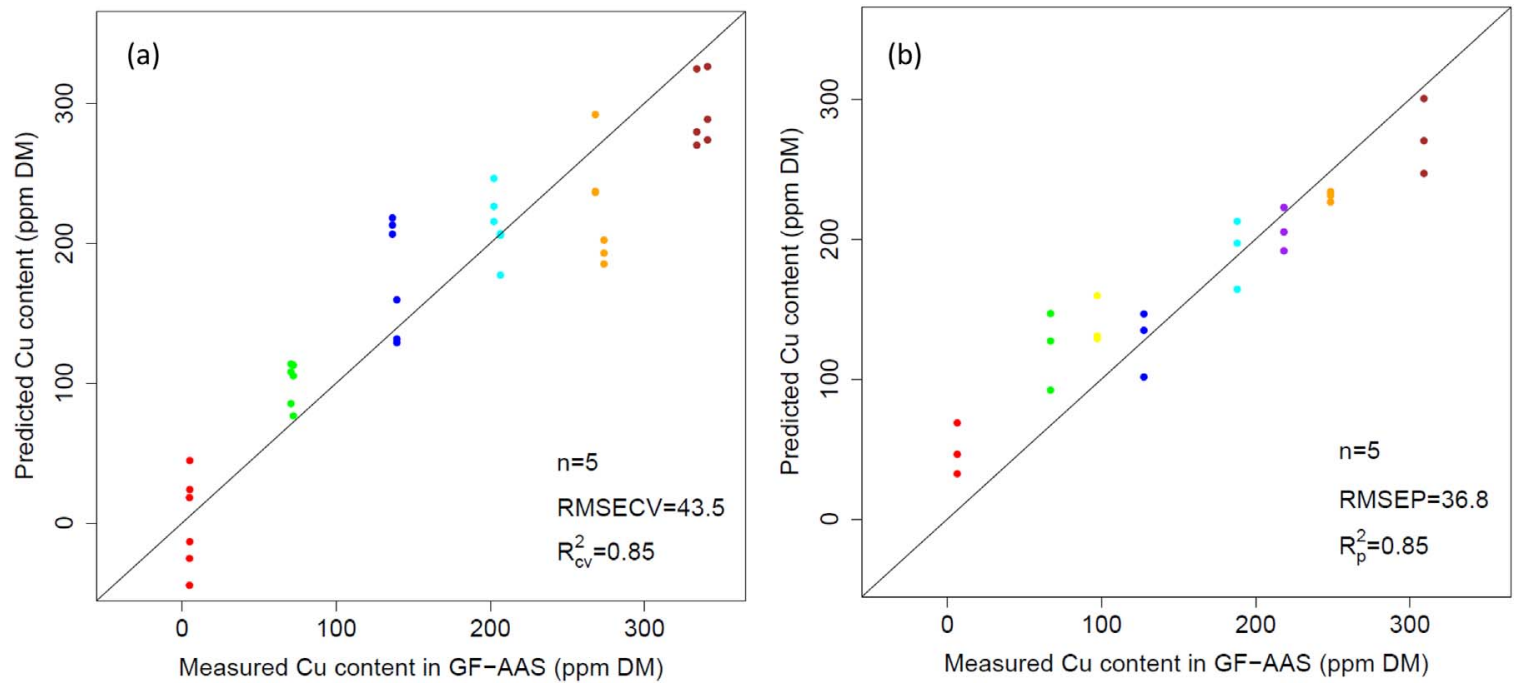

Fig. 2. Measured versus predicted Cu content (ppm DM) curves for: (a) PLS calibration model for cross-validation of batch 1 and 2, (b) PLS validation model of batch 3.

along with their elemental reference values extracted from GF-AAS analysis to develop the calibration model. Fig. 2(a) shows the crossvalidation curve of the developed PLSR model for calibration, containing the RMSECV and $R_{c v}^{2}$ along with the number of PLS components used. The optimum number of PLS components chosen was 5 based on a low RMSECV in combination with a high $R_{c v}^{2}$. The model for copper content showed a good fit, indicated by a high $R_{c v}^{2}$ of 0.85 . A RMSECV of $43.5 \mathrm{ppm}$ in dry matter (DM) indicated the deviation of the model 


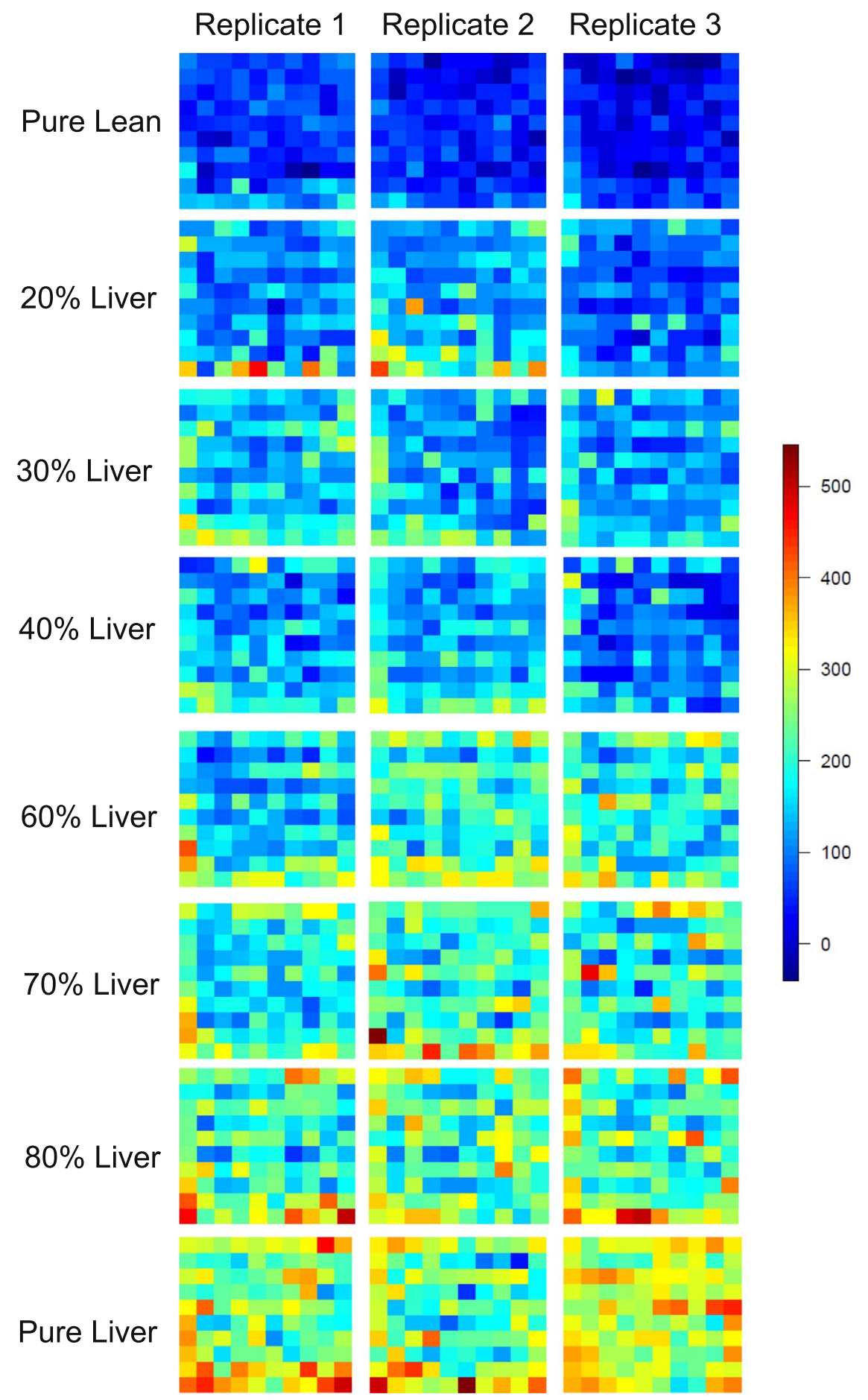

Fig. 3. Mineral mapping distribution of predicted copper content of batch 3. The colour scale indicates the copper content in ppm of DM. (For interpretation of the references to colour in this figure legend, the reader is referred to the web version of this article.)

from their reference values. The LOD for pseudounivariate calibration curves was calculated following a new approach in multivariate calibration, taking into account IUPAC official recommendations, obtaining an estimated LOD of $132 \mathrm{ppm}$. This relatively high value could be attributed to the variability in emission intensities among sample replicates as well as variability due to the use of two independent batches to build the calibration model. At the same time, factors such as chemical composition, sample-to-lens distances, particle size and homogeneity of the sample surface could also have an important role in affecting relative intensities of emission lines [33]. Nevertheless, to the best knowledge of the authors, no other studies on
LIBS analysis of meat have been conducted before using independent batches as part of the calibration and validation models $[9,28]$.

\subsubsection{Model validation}

In order to evaluate the performance of the developed PLSR model, a model validation for an independent set of experiments is required in order to ensure it will perform efficiently for similar data [18]. The data obtained for batch 3 was used as a validation set. Fig. 2(b) shows the validation curve of the developed PLSR model for prediction, containing the RMSEP and $R_{p}^{2}$. The model showed good prediction accuracy as 
(a)

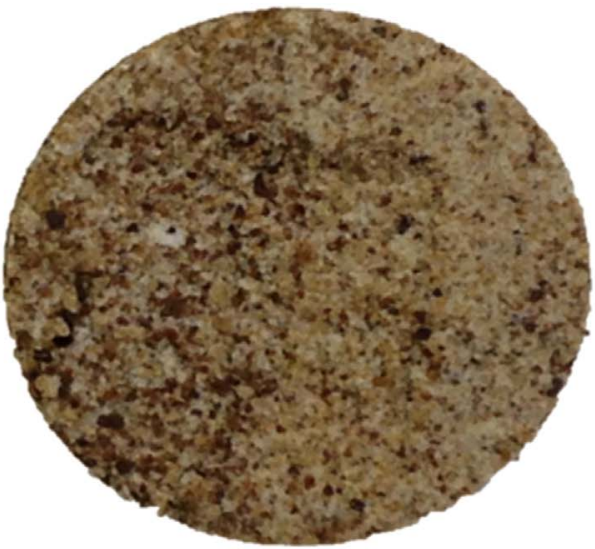

(c)

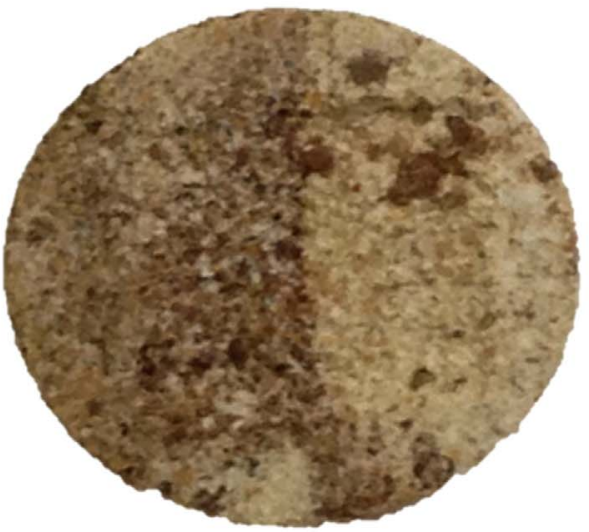

(b)
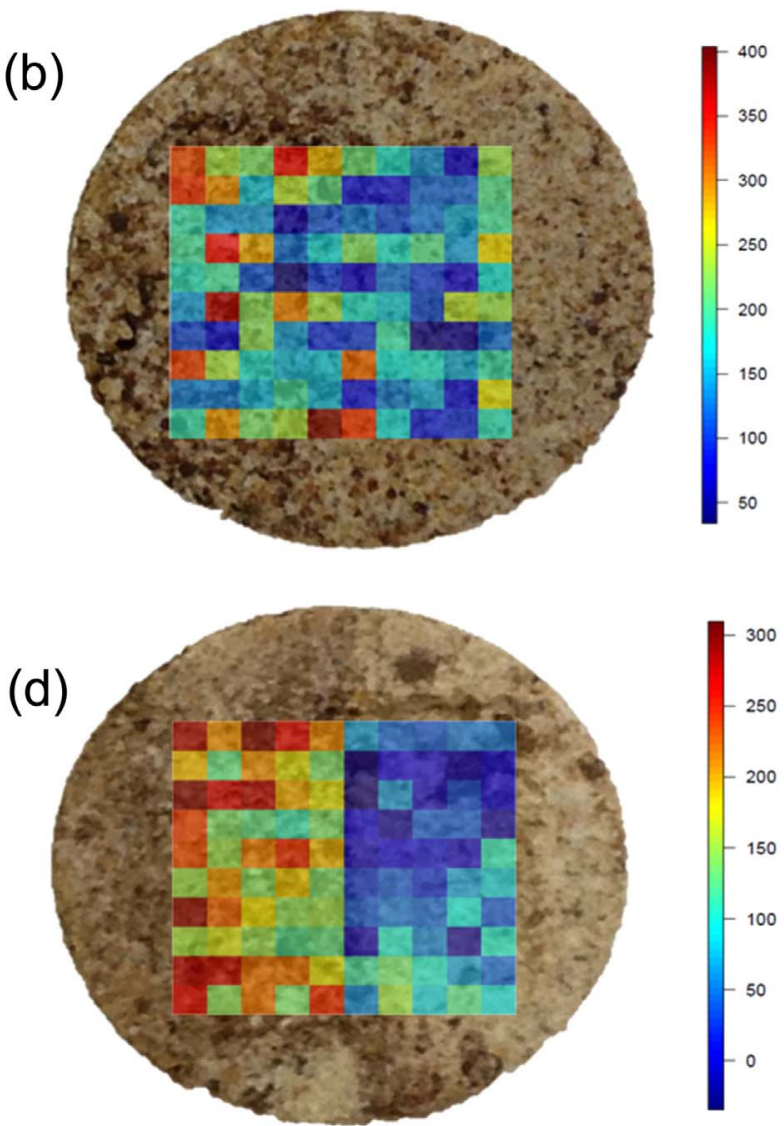

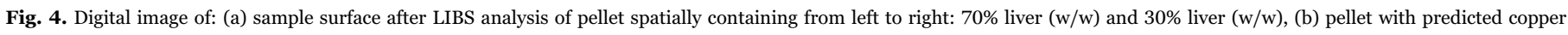

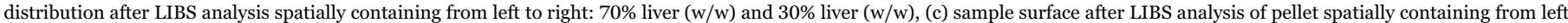

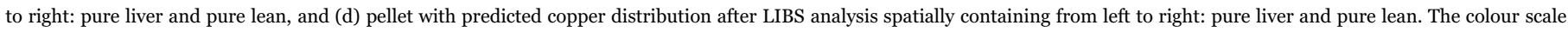
indicates the copper content in ppm of DM. (For interpretation of the references to colour in this figure legend, the reader is referred to the web version of this article.)

indicated by a high $R_{p}^{2}$ of 0.85 . A RMSEP of 36.8 (ppm DM) was obtained. A lower RMSEP when compared to the RMSECV obtained could be attributed due to the analysis of one independent batch to build the validation model as opposed to the two independent batches used in the calibration model. As previously mentioned, other factors like variability in emission intensities among sample replicates, chemical composition, sample-to-lens distances, particle size and homogeneity of the sample surface could also contribute towards the RMSEP. Overall, the performance of the PLSR shows that the models were able to quantify copper content with good accuracy, therefore being able to indirectly detect liver adulteration in beef based on its copper content.

\subsubsection{Chemical mapping}

Chemical mapping of the pellets analysed was investigated for two main objectives; Firstly, to check whether the samples were well homogenised, therefore helping to build a robust model as the spectral data obtained per pellet would be quite uniform throghout the different locations and consistent with their reference values. Secondly, to prove the ability of LIBS in combination with a motorised sample chamber to distinguish different features within a sample and therefore showing its potential for analysis in heterogenous samples.

A mineral mapping distribution of the predicted copper content (ppm DM) in 100 locations analysed per pellet of batch 3 is presented in Fig. 3. A clear distinction between the different mixtures of liver percentages is observed based on their copper content, reinforcing the potential of LIBS as a future quality monitoring tool for the meat sector. Fig. 3 also shows that the samples were relatively homogeneous with copper content consistent between replicates and evenly distributed, which contributed to a robust calibration and validation model.
To further evaluate the spatial capabilities of LIBS, two additional pellets spatially containing two different mixtures of liver content were analysed. Fig. 4(a) shows the sample surface of a pellet spatially occupying two different copper contents at $30 \%$ and $70 \%$ liver content (w/w) after LIBS analysis followed by its predicted copper distribution (ppm DM) in Fig. 4(b). Similarly, Fig. 4(c) shows the sample surface of a pellet spatially occupying two different copper contents at $0 \%$ and at $100 \%$ liver content (w/w) after LIBS analysis followed by its predicted copper distribution (ppm DM) in Fig. 4(d). As Fig. 4(b) and (d) shows, LIBS was able to successfully spatially distinguish copper content within the pellets at different ranges as indicated by colour changes from blue for low copper concentrations to red for high copper concentrations. The results presented show the suitability of LIBS to provide spatial information and therefore potential to be used on heterogeneous samples.

\section{Conclusions}

In this study, LIBS was successfully employed for quantitative analysis of copper content in minced beef samples spiked with beef liver. PLSR was performed to build a calibration and validation model. A calibration model with a high $R_{c v}^{2}$ of 0.85 and a RMSECV of $43.5 \mathrm{ppm}$ was obtained, confirming a good fit for the model. The validation model showed a good prediction accuracy with a high $R_{p}^{2}$ of 0.85 and RMSEP of $36.8 \mathrm{ppm}$. Although the $R^{2}$ obtained in both calibration and validation models would not be considered good for quantification in standard traditional analytical techniques, these are considered reasonable values for LIBS as the main feature of the technique is based on the rapid analysis and simplicity in sample preparation. The results demonstrate that LIBS offers the potential to detect and quantify liver 
adulteration in beef by indirectly measuring its copper content, as well as it offers the benefit of multi-element quantification of meat. Furthermore, although beef liver contains a richer source of copper amongst beef offal ( $98 \mathrm{ppm}$ in raw beef liver), copper content in other beef meat organs are still found in higher concentrations than in lean beef $(4.0 \mathrm{ppm}$ in raw heart and $4.3 \mathrm{ppm}$ in raw kidney as compared to $0.8 \mathrm{ppm}$ found in raw lean beef) [34]. Therefore, copper could be chosen as an indicator for lean authenticity provided that the LIBS system used is equipped with the right configuration to detect at those levels. For instance, Cho et. al. [35] determined copper amongst other trace elements in starch-based flours using a Nd:YAG laser $(1064 \mathrm{~nm})$ in pelletised samples in an argon atmosphere and reduced pressure (0.2-40 Torr). The limit of detection (LOD) for copper was established to be $1 \mathrm{ppm}$ with a relative standard deviation (RSD) of $5-8 \%$. A similar configuration could therefore be used in a future work in order to detect offal adulteration in beef, based on its copper content.

Moreover, LIBS was able to successfully distinguish spatially different copper contents within a pellet, indicating the suitability of LIBS to provide spatial information and therefore potential use on heterogeneous samples. However, further improvements may be required in order to make LIBS a suitable technique for routine analysis in an industrial environment. Overall, it can be concluded that LIBS combined with chemometrics demonstrates potential as a quality monitoring tool for the meat processing industry.

\section{Acknowledgements}

The authors would like to acknowledge funding from the Food Institutional Research Measure administered by the Department of Agriculture, Food and the Marine, Ireland (Grant agreement: 13/F/ 508).

\section{References}

[1] G. Lombardi-Boccia, S. Lanzi, A. Aguzzi, Aspects of meat quality: trace elements and B vitamins in raw and cooked meats, J. Food Compos. Anal. 18 (1) (2005) 39-46.

[2] B. Caballero, P. Finglas, F. Toldrá, Encyclopedia of Food and Health, Elsevier Science, 2015

[3] L. Prashanth, K. Kattapagari, R. Chitturi, V. Baddam, L. Prasad, A review on role of essential trace elements in health and disease, J. Dr. NTR Univ. Health Sci. 4 (2) (2015) 75-85.

[4] FAO, FAO's Animal Production and Health Division: Meat \& Meat Products. 〈http://www.fao.org/ag/againfo/themes/en/meat/background.html〉, 2016 (accessed 12.12.16)

[5] N.Z. Ballin, Authentication of meat and meat products, Meat Sci. 86 (3) (2010) $577-587$.

[6] Y. Dixit, M.P. Casado-Gavalda, R. Cama-Moncunill, X. Cama-Moncunill, P.J. Cullen, C. Sullivan, Prediction of beef fat content simultaneously under static and motion conditions using near infrared spectroscopy, J. Infrared Spectrosc. 24 (4) (2016) 353-361.

[7] Y. Dixit, M.P. Casado-Gavalda, R. Cama-Moncunill, X. Cama-Moncunill, F. Jacoby, P.J. Cullen, C. Sullivan, Multipoint NIR spectrometry and collimated light for predicting the composition of meat samples with high standoff distances, J. Food Eng. 175 (2016) 58-64.

[8] A.I. Ropodi, E.Z. Panagou, G.-J.E. Nychas, Multispectral imaging (MSI): promising method for the detection of minced beef adulteration with horsemeat, Food Control (2016).

[9] M.-B.S. Andersen, J. Frydenvang, P. Henckel, Å. Rinnan, The potential of laserinduced breakdown spectroscopy for industrial at-line monitoring of calcium content in comminuted poultry meat, Food Control 64 (2016) 226-233.

[10] L.J. Radziemski, D.A. Cremers, Handbook of Laser Induced Breakdown Spectroscopy, John Wiley \& Sons, West Sussex, England, 2006.

[11] S. Moncayo, J.D. Rosales, R. Izquierdo-Hornillos, J. Anzano, J.O. Caceres, Classification of red wine based on its protected designation of origin (PDO) using
Laser-induced Breakdown Spectroscopy (LIBS), Talanta 158 (2016) 185-191.

[12] Y.G.M. Kongbonga, H. Ghalila, M.B. Onana, Z.B. Lakhdar, Classification of vegetable oils based on their concentration of saturated fatty acids using laser induced breakdown spectroscopy (LIBS), Food Chem. 147 (2014) 327-331.

[13] G. Bilge, H.M. Velioglu, B. Sezer, K.E. Eseller, I.H. Boyaci, Identification of meat species by using laser-induced breakdown spectroscopy, Meat Sci. 119 (2016) $118-122$.

[14] M.A. Gondal, Y.B. Habibullah, U. Baig, L.E. Oloore, Direct spectral analysis of tea samples using $266 \mathrm{~nm}$ UV pulsed laser-induced breakdown spectroscopy and cross validation of LIBS results with ICP-MS, Talanta 152 (2016) 341-352.

[15] Q. Sun, M. Tran, B.W. Smith, J.D. Winefordner, Direct determination of P, Al, Ca, $\mathrm{Cu}, \mathrm{Mn}, \mathrm{Zn}, \mathrm{Mg}$ and $\mathrm{Fe}$ in plant materials by laser-induced plasma spectroscopy, Can. J. Anal. Sci. Spectrosc. 44 (6) (1999) 164-170.

[16] T.-L, Zhang, W. Shan, T. Hong-Sheng, W. Kang, D. Yi-Xiang, L. Hua, Progress of chemometrics in Laser-induced breakdown spectroscopy analysis, Chin. J. Anal. Chem. 43 (6) (2015) 939-948.

[17] K.A. Bakeev, Process analytical technology: spectroscopic tools and implementation strategies for the chemical and pharmaceutical industries, John Wiley \& Sons, 2010.

[18] G. ElMasry, D.-W. Sun, P. Allen, Chemical-free assessment and mapping of major constituents in beef using hyperspectral imaging, J. Food Eng. 117 (2) (2013) $235-246$.

[19] N. Morsy, D.W. Sun, Robust linear and non-linear models of NIR spectroscopy for detection and quantification of adulterants in fresh and frozen-thawed minced beef, Meat. Sci. 93 (2) (2013) 292-302.

[20] L.C. Peruchi, L.C. Nunes, G.G.A. de Carvalho, M.B.B. Guerra, E. de Almeida, I.A. Rufini, D. Santos Jr, F.J. Krug, Determination of inorganic nutrients in wheat flour by laser-induced breakdown spectroscopy and energy dispersive X-ray fluorescence spectrometry, Spectrochim. Acta Part B: At. Spectrosc. 100 (2014) $129-136$.

[21] D.W. Hahn, N. Omenetto, Laser-Induced Breakdown Spectroscopy (LIBS), Part I: Review of Basic Diagnostics and Plasma-Particle Interactions: Still-Challenging Issues within the Analytical Plasma Community, Appl. Spectrosc. 64 (12) (2010) 335A-336A.

[22] R. Core Team, R: A language and environment for statistical computing. $\mathrm{R}$ Foundation for Statistical Computing, Vienna, Austria, 2012, ISBN 3-900051-07-0, 2014.

[23] B.-H. Mevik, R. Wehrens, K.H. Liland, pls: Partial least squares and principal component regression, R. Package Version 2.4-3 (2013).

[24] Å. Rinnan, F. van den Berg, S.B. Engelsen, Review of the most common preprocessing techniques for near-infrared spectra, TrAC Trends Anal. Chem. 28 (10) (2009) 1201-1222.

[25] T.-L. Zhang, S. Wu, H.-S. Tang, K. Wang, Y.-X. Duan, H. Li, Progress of Chemometrics in Laser-induced Breakdown Spectroscopy Analysis, Chin. J. Anal. Chem. 43 (6) (2015) 939-948.

[26] D. Syvilay, N. Wilkie-Chancellier, B. Trichereau, A. Texier, L. Martinez, S. Serfaty, V. Detalle, Evaluation of the standard normal variate method for Laser-Induced Breakdown Spectroscopy data treatment applied to the discrimination of painting layers, Spectrochim. Acta Part B: At. Spectrosc. 114 (2015) 38-45.

[27] A. Kramida, Y. Ralchenko, J. Reader, N.A. Team, NIST Atomic Spectra Database (Ver. 5.4) (Online), National Institute of Standards and Technology, Gaithersburg, MD, 2016.

[28] G. Bilge, B. Sezer, K.E. Eseller, H. Berberoglu, A. Topcu, I.H. Boyaci, Determination of whey adulteration in milk powder by using laser induced breakdown spectroscopy, Food Chem. 212 (2016) 183-188.

[29] R. Cama-Moncunill, M. Markiewicz-Keszycka, Y. Dixit, X. Cama-Moncunill, M.P. Casado-Gavalda, P.J. Cullen, C. Sullivan, Multipoint NIR spectroscopy for gross composition analysis of powdered infant formula under various motion conditions, Talanta 154 (2016) 423-430.

[30] F. Tao, Y. Peng, C.L. Gomes, K. Chao, J. Qin, A comparative study for improving prediction of total viable count in beef based on hyperspectral scattering characteristics, J. Food Eng. 162 (2015) 38-47.

[31] F. Allegrini, A.C. Olivieri, IUPAC-Consistent Approach to the Limit of Detection in Partial Least-Squares Calibration, Anal. Chem. 86 (15) (2014) 7858-7866.

[32] C.A. D’Angelo, M. Garcimuño, D.M. Díaz Pace, G. Bertuccelli, Plasma diagnostics from self-absorbed doublet lines in laser-induced breakdown spectroscopy, J. Quant. Spectrosc. Radiat. Transf. 164 (2015) 89-96.

[33] F.J. Fortes, J. Moros, P. Lucena, L.M. Cabalín, J.J. Laserna, Laser-induced breakdown spectroscopy, Anal. Chem. 85 (2) (2012) 640-669.

[34] USDA National Nutrient Database for Standard Reference, US Department of Agriculture, Agricultural Research Service, Nutrient Data Laboratory, 2015.

[35] H.-H. Cho, Y.-J. Kim, Y.-S. Jo, K. Kitagawa, N. Arai, Y.-I. Lee, Application of laserinduced breakdown spectrometry for direct determination of trace elements in starch-based flours, J. Anal. At. Spectrom. 16 (6) (2001) 622-627. 